\title{
Acoustic Impulses Generated by Air-Bubble Stimulated Underwater Spark Discharges
}

\author{
Y. Sun, I. V. Timoshkin, M. J. Given, M. P. Wilson, T. Wang and S. J. MacGregor \\ Department of Electronic and Electrical Engineering \\ University of Strathclyde \\ Glasgow, United Kingdom \\ N. Bonifaci \\ CNRS, G2E Laboratory \\ Grenoble, France
}

\begin{abstract}
Considerable research efforts have been expended in investigating and optimising the emission of acoustic pressure impulses by high power spark discharges in water. Several methods are typically used to generate transient underwater spark discharges, including breakdown of water by stressing electrode(s) with $\mathrm{HV}$ impulses, initiation of spark discharges with thin metallic wires placed between opposite electrodes and by injecting gas bubbles into the inter-electrode gap. This paper is aimed at the investigation of the acoustic, hydrodynamic and electrical characteristics of underwater spark discharges initiated by injected air-bubbles. At a constant applied voltage, the injection of air bubbles allows the inter-electrode gap to be increased thus producing stronger acoustic impulses. This paper reports on the impact of injected air bubbles on the characteristics of transient underwater plasma discharges and on the functional relationships between hydrodynamic and electrical parameters of such discharges, including breakdown voltage, plasma resistance, energy delivered into the plasma cavity and the period of gas/plasma cavity oscillation. An analytical model which relates the acoustic efficiency, plasma resistance and energy available in discharge has been developed which can be used for the optimisation and tailoring of underwater plasmaacoustic sources.
\end{abstract}

Index Terms - Underwater spark discharges, acoustic emission, hydrodynamics.

\section{INTRODUCTION}

AN underwater spark discharge (UWSD) formed between high voltage and ground electrodes generates an acoustic pressure impulse, which quickly becomes an acoustic wave that propagates away from the breakdown channel. The high degree of control, straightforward optimisation and tailoring of the emitted acoustic pressure impulses make plasma-acoustic sources and UWSD's an attractive solution for different technological applications. For example, pressure impulses generated by the UWSD's are used in material recycling and mineral processing [1], in water treatment operations, [2, 3], and in ultra-wideband sonar technology, [4].

Recently, a number of comprehensive studies characterising UWSD's and their associated pressure impulses have been conducted, [5-10]. It was found that the peak magnitude of the acoustic impulses strongly depends on the inter-electrode gap, i.e. an increase in the inter-electrode distance results in generation of stronger acoustic pressure

Manuscript received on 22 December 2017, in final form 31 May 2018, accepted 3 June 2018. Corresponding author: Y. Sun. impulses, $[5,7,8,9]$. However, when using water with an elevated electrical conductivity (as compared with that of distilled water), for example in the case of UWSD's in tap water or sea water, the ionic conduction may cause a significant pre-breakdown drop in the voltage across the electrodes. In the case of free, un-triggered discharges in water with conductivity of $66 \mu \mathrm{S} / \mathrm{cm}$ the maximum inter-electrode distance, which was possible to break with $35 \mathrm{kV}$ impulses, was $15 \mathrm{~mm}$, [6]. It was not possible to achieve repeatable spark discharges in water with such conductivity with further increases in the inter-electrode gap. An increase in the applied voltage may help to achieve spark discharges in longer gaps, however in many technological applications it may not be practical from the point of view of insulation coordination.

The initial, pre-breakdown, stage of the development of a spark discharge in a liquid includes multiple complex processes, such as charge injection and field modification. A comprehensive study of the charge injection process in water and water/glycol solutions was conducted in [11-13], where it was shown that the injected charge modifies the electric field in the liquid near the electrodes, and can result in higher breakdown voltage and faster pre-breakdown voltage decay. 
Detailed analysis of such processes is beyond the scope of the present paper, since it is focused on the post-breakdown characteristics of underwater spark discharges.

As reported in $[8,10]$, the use of a thin conductive wire placed between the electrodes allows the distance between the electrodes to be increased. However, this approach requires replacement of the initiating wire after each shot, thus such systems typically operate in a single shot/low frequency pulse regime. To increase the operating frequency and to minimise pre-breakdown ionic losses, gas bubbles can be injected into the inter-electrode gap. The injected gas bubbles help to reduce both the time to breakdown and the breakdown voltage, due to the lower breakdown strength of gases (as compared to that of the water), and the field enhancement within the bubbles (due to the permittivity mismatch between the water and the gas).

In [14], different gases ( $\mathrm{Ar}, \mathrm{CO}_{2}, \mathrm{CH}_{4}$, and $\mathrm{C}_{3} \mathrm{H}_{8}$ ) were used to form bubbles in distilled water to stimulate spark discharges and to keep the breakdown voltage at a relatively low level. In [15] nitrogen bubbles were injected between parallel-plane electrodes to facilitate the development of spark discharges. In [16], the influence of the dimension of the injected gas bubbles on the breakdown initiation process was studied. In $[17,18]$ the role of gas bubbles in the breakdown initiation process in water has been analysed. Thus, the injection of gas bubbles may provide a practical solution for triggering spark underwater spark discharges in longer gaps in water with elevated electrical conductivity.

In the present paper, air bubbles were injected into the inter-electrode gap to facilitate development of the transient (spark) plasma channels. This study is aimed at a comprehensive investigation of the hydrodynamic, acoustic and electrical parameters of air-bubble-stimulated UWSD's, including the acoustic magnitude, the period of cavity oscillation, the breakdown voltage, the plasma resistance and the energy delivered into the plasma.

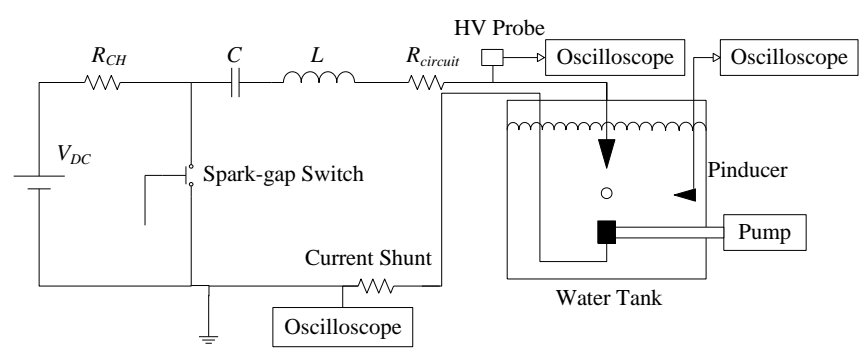

Figure 1. Schematic diagram of the pulse power system and the electrodes topology with an external pump: $V_{D C}$, DC generator; $R_{C H}$, charging resistance; $C$, energy storage capacitance; $R_{\text {circuit }}$, circuit resistance; $L$, circuit inductance.

An analytical model has been developed based on Gilmore's bubble oscillation model $[5,6]$, to obtain the timedependent radius of the vapour/gas cavity formed by the spark discharge and the peak magnitude of the acoustic impulse generated by this transient cavity. The acoustic efficiency of UWSD's has been obtained as a function of their electrical parameters (the constant plasma resistance and the energy delivered into the plasma). The obtained functional dependencies of the hydrodynamic and electrical parameters of the UWSD's can be used for optimisation of the plasmaacoustic systems and tailoring their acoustic emission parameters for specific practical applications.

\section{EXPERIMENTAL SET-UP}

The experimental setup used in this work included an impulsive voltage generation system, a pair of electrodes with controllable separation and an external air pumping system. The air pump was used to inject a continuous flow of air bubbles into the inter-electrode gap. A schematic diagram of the setup is shown in Figure 1. The impulsive voltage generation system used in these tests was the same as the pulsed power system described in [7, 8]. It consists of a Glassman high voltage DC power supply (series EH), used to charge different combinations of HV capacitors from Maxwell Laboratories Ltd, USA. The pulsed power system is triggered by a manually controlled spark-gap switch.

The stainless steel electrodes were orientated vertically in the center of a large water tank filled with tap water, with dimensions of $2 \mathrm{~m}$ (length) $\times 1 \mathrm{~m}$ (width) $\times 1 \mathrm{~m}$ (height). The high voltage electrode was in a shape of cone with a $5 \mathrm{~mm}$ base radius and a tip radius of $\sim 0.8 \mathrm{~mm}$ in order to enhance the electrical field in its vicinity. The lower, ground electrode was cylindrical in shape with a diameter of $15 \mathrm{~mm}$, and had an embedded air flow channel. The air bubbles were injected into the inter-electrode gap through a $1 \mathrm{~mm}$ aperture of the gas channel formed in the lower, ground electrode, and the topology of this electrode is shown in Figure 2. Air was injected into the gas channel by an aquarium pump; the air flow in the system being controlled by gas regulators. The pump was kept on during the whole period of testing to generate a consistent flow of air-bubbles between the electrodes from shot to shot. The injected air bubbles will move vertically up, towards the upper conical electrode.

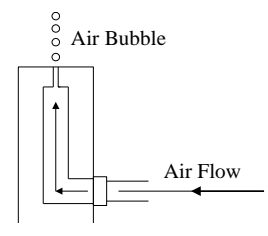

Figure 2. Ground electrode modified with air flow channel.

The transient voltage and current waveforms were obtained using a PVM-5 high voltage probe (North Star Ltd USA, with the following nominal parameters: 1000:1 division ratio, $80 \mathrm{MHz}$ bandwidth, $400 \mathrm{M} \Omega$ input resistance, and $12 \mathrm{pF}$ input capacitance, [19]) and a current shunt with the resistance of $29.6 \mathrm{~m} \Omega$, (Samtech Ltd, Scotland). These waveforms were recorded using a Tektronix 2054B digitising oscilloscope (500 MHz bandwidth, $2 \mathrm{GS} / \mathrm{s}$ sampling rate). The acoustic signals were obtained by using a piezo-electric acoustic sensor, Pinducer VP1093 (Valpey Fisher Ltd USA) and were recorded by a Tektronix 5034B digitising oscilloscope (350 $\mathrm{MHz}$ bandwidth, $5 \mathrm{GS} / \mathrm{s}$ sampling rate). Two different time bases were used to optimise the resolution of the acoustic signals emitted by the expanding and collapsing gas cavities. 
The magnitude of the acoustic impulse was captured with a 200 ns/division time-base, while two consecutive acoustic signals produced by the expanding and collapsing cavities were obtained with a $2 \mathrm{~ms} /$ division time-scale in order to register the period of cavity oscillation.

\section{RESULTS}

A comprehensive investigation of the acoustic and hydrodynamic characteristics of the air-bubble-stimulated UWSD's was conducted. The relationships established between the acoustic, hydrodynamic and electrical parameters are presented and discussed in this section. The acoustic and hydrodynamic parameters include the peak acoustic magnitude, $P_{a c-m a x}$ and the period of the cavity's primary oscillation, $T_{c a v}$. The electrical parameters are the breakdown voltage, $V_{b r}$, the discharged current, $I$, the plasma resistance, $R_{p l}$, and the energy delivered into plasma, $E_{p l}$.

Different combinations of capacitance and charging voltages were used in the present work. Three values of energy-storage capacitance in the pulsed power circuit, $C$, were used: 155, 266 and $533 \mathrm{nF}$. The charging voltage levels, $V_{c h}$, were $25 \mathrm{kV}, 30 \mathrm{kV}$ and $35 \mathrm{kV}$. These levels of $C$ and $V_{c h}$ were chosen to provide a nominal stored energy, $0.5 C V_{c h}{ }^{2}$, in the range from $\sim 48 \mathrm{~J}$ to $\sim 326 \mathrm{~J}$, while keeping charging voltages below $40 \mathrm{kV}$. Similar energies and charging voltage levels were used in several of the practical applications discussed in $[1,6,20]$. The charging voltages, capacitances and energies used in the present work are also close to those used in previous studies focused on free and wire-guided discharges in water, [5-8]. Thus, the results obtained in the present paper, for air-bubble stimulated underwater spark discharges, can be compared with previously-published data on the acoustic, hydrodynamic and electrical parameters of free and wire-guided underwater spark discharges.

Three inter-electrode distances, $\ell,(15 \mathrm{~mm}, 20 \mathrm{~mm}$ and $25 \mathrm{~mm}$ ) were chosen for this study to allow comparison of the results obtained for the air-bubble-stimulated discharges with the results for free underwater spark discharges obtained for shorter gaps, $\ell<15 \mathrm{~mm}$, in [7]. However, when using the $155 \mathrm{nF}$ capacitance the success rate of spark breakdown events in long, $20 \mathrm{~mm}$ and $25 \mathrm{~mm}$, gaps in tap water with conductivity of $66 \mu \mathrm{S} / \mathrm{cm}$ was low, less than $5 \%$. Therefore, the acoustic and hydrodynamic data for $C=155 \mathrm{nF}$ were obtained only for $\ell=15 \mathrm{~mm}$.

\subsection{ELECTRICAL AND ACOUSTIC WAVEFORMS}

By applying a HV impulse to a pair of electrodes in the water tank, breakdown can be initiated by injection of air bubbles into the inter-electrode gap resulting in formation of a low resistance plasma channel between the electrodes. Examples of typical post-breakdown voltage and current waveforms obtained for the air-bubble-stimulated discharges are shown in Figure 3. In Figure 3(a), the breakdown voltage, $V_{b r}$, is read as $-33.8 \mathrm{kV}$ at the moment when the voltage starts to drop due to the formation of the plasma channel. At the same time, a conductive current appears in the circuit and under-damped current and voltage oscillations are observed in both waveforms.

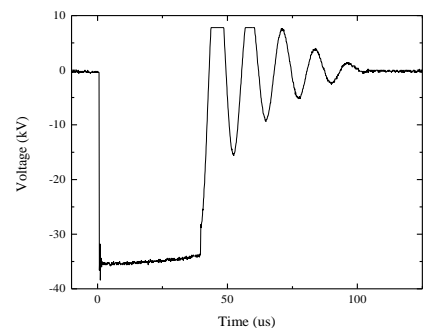

(a)

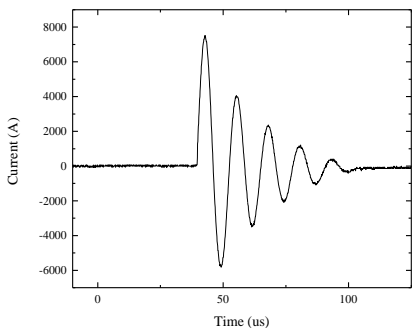

(b)
Figure 3. The example electrical waveforms: (a) voltage; (b) current, obtained from a discharge achieved in a $25 \mathrm{~mm}$ gap with $533 \mathrm{nF}$ capacitance.

The profiles of the waveforms in Figure 3 are similar to those obtained in [7] for free spark discharges in water. In [7, 8], the general solution for the post-breakdown $R-L-C$ circuit has been successfully verified to describe the dynamic oscillations of the circuit current:

$$
I(t)=I_{0} e^{-\alpha t} \sin (\omega t)
$$

where $I_{0}$ is a constant related to the peak current, $\alpha$ is the attenuation coefficient and $\omega$ is the angular frequency of the oscillations.

This approach assumes a constant plasma resistance. It is known that the resistance of the underwater spark channel significantly reduces during the first quarter-cycle of the oscillating current from a few $\Omega$ s to a few $m \Omega s$, [5]. The spark resistance remains in this range until the end of the first halfcycle of the oscillating discharge current. An equivalent constant value of resistance obtained from the oscillating current wave form can be used to represent the energy delivered to the spark channel. In [5] the validity of the constant plasma resistance approximation in the hydrodynamic modelling of underwater spark discharges was confirmed, and this approach was adopted in the present study.

The total resistance of the circuit, $R_{\text {total }}$, which includes the plasma channel resistance can be obtained by fitting the experimental current wave forms with equation (1). $R_{\text {total }}$ includes all resistive elements in the pulsed power circuits and the resistance of the plasma channel, $R_{p l}$. Therefore, $R_{p l}$, is calculated as the difference between $R_{\text {total }}$ and the resistance of the pulsed power driving circuit, $R_{\text {circuit }}$ :

$$
R_{p l}=R_{\text {total }}-R_{\text {circuit }}
$$

Based on the behavior of the circuit, at equivalent current levels, when the gap is short-circuited, $\left(R_{p l}=0\right), R_{\text {circuit }}$, can be obtained. One the plasma resistance is known, the electrical energy delivered into the plasma channel, $E_{p l}$, can then be calculated:

$$
E_{p l}=R_{p l} \int I(t)^{2} d t
$$

Typical acoustic signals generated by an air-bubblestimulated UWSD's are shown in Figure 4. Figure 4(a) shows the profile of the primary acoustic impulse, $P_{a c}(t)$, registered 
with a $200 \mathrm{~ns} /$ division time-base. The value of the first peak in acoustic signal is used to represent the magnitude of the primary (first) acoustic impulse emitted by the spark discharge cavity, $P_{\text {ac-max }}$.

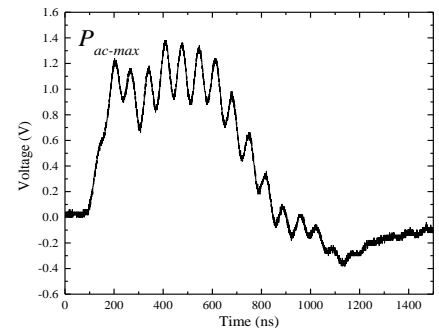

(a)

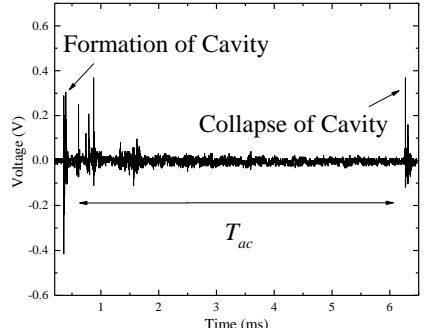

(b)
Figure 4. Acoustic signals generated by discharges in 20 and $25 \mathrm{~mm}$ gaps: (a) the profile of the acoustic magnitude; (b) the primary and secondary acoustic impulses emitted at the formation and collapse of the cavity. $P_{a c-\max }$ is the first peak of the impulse and $T_{c a v}$ indicates the time duration of the primary oscillation of the cavity.

Figure 4b shows two major (primary and secondary) acoustic impulses captured at the moments of formation and collapse of the spark discharge cavity, yielding the period of the cavity's oscillation, $T_{c a v}$, as the time interval between these two impulses. Due to the unavailability of calibration for the acoustic sensor from the manufacturer, the sensor output in Volts was used to represent the magnitude and wave-shape of the acoustic signals. In the ideal case with no energy loss, the Rayleigh solution describes the dynamic oscillation of the cavity. This solution describes well both, the expansion and collapse stages of the cavity forming a complete cavity cycle, and it links the period of such an cavity oscillation cycle, $T_{\text {cav }}$, with the maximal radius of the cavity, $r_{\max }$, [21-23]. The electrical energy transferred into the plasma is not only converted into acoustic energy and the internal energy of the plasma in the channel, but also into the energy of light emission and other potential energy losses due to the non-ideal spherical topology of the bubble, heat transfer across the plasma/gas-liquid interface, and other loss mechanisms. It was estimated that these energy losses only accounted for less than $\sim 10 \%$ of the total energy delivered into plasma, [5].

\subsection{THE ROLE OF BREAKDOWN VOLTAGE}

The period of cavity oscillation, $T_{c a v}$, and the peak magnitude of the acoustic signal, $P_{a c-\max }$, were obtained for all three inter-electrode distances under different energisation conditions. These data are shown in Figure 5 as functions of $V_{b r}$ with each point representing a single breakdown event stimulated by air bubbles.

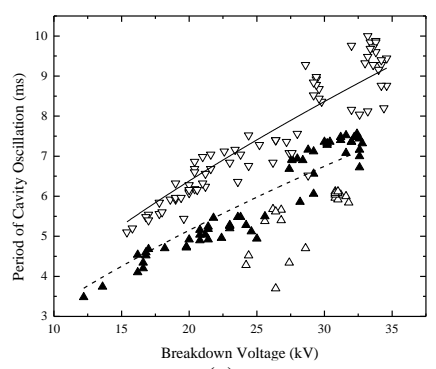

(a)

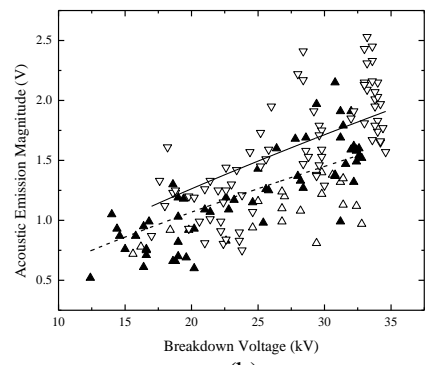

(b)
Figure 5. Relationships between: $T_{c a v}$ and $V_{b r}$, (a); $P_{a c-\max }$ and $V_{b r}$, (b). Open upper triangle represents data for $C=155 \mathrm{nF}$; closed upper triangle represents data for $C=266 \mathrm{nF}$; open inverted triangle represents data for $C=533 \mathrm{nF}$. Solid and dashed lines in (a) indicate the fitting of equation (4) and those in (b) indicate the fitting of equation (5).

It can be seen from Figure 5(a) that the period of cavity oscillation increases with breakdown voltage for all interelectrode distances and the discharges achieved with higher values of $C$ typically produce longer $T_{c a v}$ at the same breakdown voltage. Either large values of $V_{b r}$ or high $C$ in Figure 5a correspond to a larger amount of energy available in the discharge which results in longer $T_{c a v}$. In [7], a similar dependency of $T_{c a v}$ on $V_{b r}$ and $C$ was observed for free UWSD's and a phenomenological equation was provided to describe this relation:

$$
T_{c a v}=a C^{1 / 3} V_{b r}^{2 / 3}
$$

where $a$ is a phenomenological fitting coefficient, $T_{\text {cav }}$ is in ms, $C$ is in $\mu \mathrm{F}$ and $V_{b r}$ is in $\mathrm{kV}$.

Table 1. Coefficient $a$ for Equation (4)

\begin{tabular}{c|c|c}
\hline$C$ & $266 \mathrm{nF}$ & $533 \mathrm{nF}$ \\
\hline$a$ & $0.109 \pm 0.002$ & $0.107 \pm 0.002$ \\
\hline
\end{tabular}

Equation (4) was fitted to the experimental $T_{c a v}$ values obtained for the air-bubble-triggered discharges (generated with $266 \mathrm{nF}$ and $533 \mathrm{nF}$ capacitances) by using OriginLab 9.0 graphing software. No fitting for the $C=155 \mathrm{nF}$ case is provided as only a single inter-electrode distance, $\ell=15 \mathrm{~mm}$, was used for this value of $C$.

This fitting procedure provided the fitting coefficient, $a$, for the different values of $C$ as given in Table 1 ; the analytical fitting curves are shown in Figure 5a. As indicated by the solid line $(C=533 \mathrm{nF})$ and the dashed line $(C=266 \mathrm{nF})$ in Figure 5a, equation (4) demonstrates a good agreement with the experimental data. The quality of the fitting obtained by equation (4) has been observed, for free discharges in [6] and for air-bubble stimulated discharges in the present work. Thus, it confirms that the post-breakdown functional behavior of $T_{c a v}$ is not affected by the discharge initiation mechanism. It is also worthy of note that the values of the proportionality coefficient, $a$, for both 266 and $533 \mathrm{nF}$ cases are close to each other, indicating that the functional behavior of $T_{\text {cav }}$ is not sensitive to $\ell$ for free and air-bubble-stimulated discharges.

Figure 5b shows the peak magnitude of the acoustic impulse as a function of the breakdown voltage. Similar to the 
relationship established for $T_{c a v}$ and $V_{b r}, P_{a c-\max }$ increases with an increase in $V_{b r}$ for all tested capacitances and interelectrode distances; the discharges with larger $C$ 's also generate higher $P_{a c-\max }$. As compared with Figure 5a, the data in Figure 5b show a higher degree of dispersion; thus discrimination between $P_{a c-\max }$ for different values of $C$ ' $s$ is not as well defined as that for $T_{c a v}$. This is due to the higher sensitivity of $P_{a c-\max }$ to the choice of $\ell$, which was observed in [8, 10, 24]. In [24], a phenomenological relation has been established which relates the intensity of the shock wave with the plasma channel length, $\ell: P_{m}=0.58 \ell^{0.75}$. However, considering the limited range of the values of $\ell$ used in the present work, the relationship originally developed in [7] for free discharges was also used to describe $P_{a c-\max }\left(V_{b r}\right)$ in the case of air-bubble-stimulated discharges:

$$
P_{a c-\max }=b C^{\theta / 3} V_{b r}{ }^{2 \theta / 3}
$$

where $b$ is a fitting coefficient, $\theta=1.13, P_{a c-\max }$ is in $\mathrm{V}, C$ is in $\mu \mathrm{F}$, and $V_{b r}$ is in $\mathrm{kV}$.

By fitting Equation (5) to the experimental data shown in Figure 5(b) (excluding the $155 \mathrm{nF}$ case), it was found that $b=(1.36 \pm 0.06) \times 10^{-2}$ for the $266 \mathrm{nF}$ case and $b=(0.04 \pm 0.06) \times 10^{-2}$ for the $533 \mathrm{nF}$ case. The results of this fitting procedure are shown as the solid and dashed lines, in Figure 5(b). The fitting curves in Figure 5(b) show a reasonable agreement with the experimental data, confirming that equation (5) can be used to describe the relation between $P_{a c-\max }$ and $V_{b r}$ for air-bubble-stimulated discharges. This relationship can be used to predict the magnitude of the acoustic impulses generated for specific pulsed power circuit parameters.

\subsection{THE ROLE OF ENERGY}

The energy released in the plasma channel during the postbreakdown period is an important factor which can be used in the development and optimisation of the plasma-acoustic sources. By using Equations (1)-(2) together with the experimental current waveforms, the energy delivered into the plasma channel, $E_{p l}$, has been obtained for all combinations of the capacitances and inter-electrode distances.

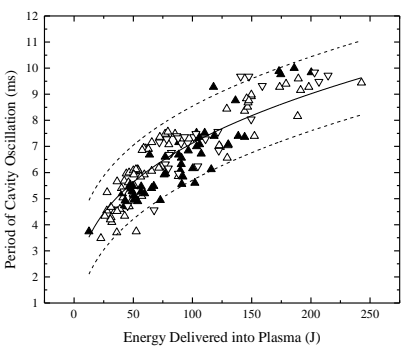

(a)

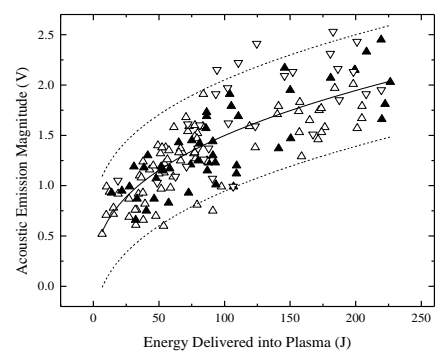

(b)
Figure 6. Relationships between: $T_{c a v}$ and $V_{b r}$, (a); $P_{a c-\max }$ and $E_{p l}$, (b). Open upper triangles represent data for $\ell=15 \mathrm{~mm}$; closed triangles represent data for $\ell=20 \mathrm{~mm}$; open inverted triangles represent data for $\ell=25 \mathrm{~mm}$. Solid line indicates the fitting of equation (6); dashed lines represent the $95 \%$ prediction intervals.
Figure 6 shows $T_{c a v}$ and $P_{a c-\max }$ as functions of the energy delivered into the plasma, $E_{p l}$, for three values of $C$. It can be seen that both, $T_{c a v}$ and $P_{a c-m a x}$ increase with an increase in $E_{p l}$. In [7], the phenomenological relations for the dependency of $T_{c a v}$ and $P_{a c-m a x}$ on $E_{p l}$ have been established for the free UWSD's. These phenomenological relationships can be applied to establish the functional behavior of $T_{c a v}$ and $P_{a c-\max }$ for air-bubble-stimulated discharges:

$$
T_{c a v}=k E_{p l}^{1 / 3}, \quad P_{a c-\max }=m E_{p l}^{\theta / 3}
$$

where $k$ and $m$ are phenomenological fitting coefficients, $T_{c a v}$ is in $\mu \mathrm{s}, P_{a c-\max }$ is in $\mathrm{V}$, and $E_{p l}$ is in $\mathrm{J}$.

The solid lines in Figure 6 show the fitting results obtained using Equations (6) and the dashed lines show the $95 \%$ prediction intervals. It can be seen that both equations (6) provide a good agreement with the experimental data with most of $T_{c a v}$ and $\mathrm{P}_{a c-\max }$ data are located within the $95 \%$ prediction range. These results confirm that the scaling relations (6) can be used in the case of air-bubble-stimulated discharges.

The proportionality coefficient, $k$, was obtained by the fitting procedure and found to be $k=1.50 \pm 0.02$ for data shown in Figure 6a. This value is close to $k=(1.6-1.7)$ obtained for free discharges in [7]. The proportionality coefficient, $m$, in equation (6) for $P_{a c-\max }$ was found to be $m=0.26 \pm 0.01$. The importance of equations (6) is not only in providing the effective prediction of the acoustic and hydrodynamic characteristics of UWSD's but also in helping to achieve specific properties of the acoustic emission by adjusting and optimising appropriate parameters of the pulsed power circuit.

In the present work, only the energy released into the postbreakdown plasma channel was considered; this energy defines the hydrodynamic and acoustic parameters of the spark breakdown gas/plasma cavities studied in this paper. The role of the energy in the pre-breakdown processes in insulating liquids is discussed in [25 - 27]. It was shown that the energy stored electrostatically in the electrode gap, or in an additional capacitor connected in parallel with the electrodes, may affect the gap breakdown voltage; in [27], it was postulated that this locally-stored energy permits "weaker initiating events to grow to a breakdown point". However, the capacitance of the electrode system used in the present work is in the range from $\sim 14 \mathrm{pF}$ (for a $15 \mathrm{~mm}$ gap) to $\sim 30 \mathrm{pF}$ (for a $25 \mathrm{~mm}$ gap), and the nominal capacitance of the HV probe connected in parallel with the water gap is $12 \mathrm{pF}$. These values are significantly lower than the energy-storage capacitances, 155, 266 and $533 \mathrm{nF}$. Therefore, in the experimental arrangements used herein, the energy stored electrostatically in the water gap is negligible compared to the energy stored in the capacitor(s) of the pulse-driving circuit. Thus, it can be assumed that the energy stored in the water gap does not significantly affect either the breakdown or post-breakdown characteristics of the underwater spark discharges studied in the present work. 


\subsection{ACOUSTIC EFFICIENCY}

The acoustic energy, $E_{a c}$, indicates the amount of energy related to the pressure impulse generated by the formation of the gas-plasma cavity after breakdown. In this work, $E_{a c}$ refers to the acoustic energy associated with the primary acoustic impulse (the first acoustic impulse emitted by the discharge cavity). The acoustic wave at a distance, $d$, which is significantly longer that the inter-electrode gap, $d \gg \ell$, can be considered as a spherical wave and $E_{a c}$ can be calculated as:

$$
E_{a c}=4 \pi Z_{a c}^{-1} d^{2} \int P_{a c}(t)^{2} d t
$$

where $P_{a c}(t)$ is the time-dependent waveform of the acoustic pressure impulse at the specific observation point, $d ; Z_{a c}$ is the acoustic impedance of water (defined as $Z_{a c}=\rho_{0} c_{0}$ where $\rho_{0}$ is the density of water, and $c_{0}$ is the local speed of sound in water). The energy losses during the acoustic impulse's propagation are neglected in this analysis, thus $E_{a c}$ obtained at $d$ represents the initial acoustic energy transferred from the energy delivered into the plasma, $E_{p l}$ into the acoustic impulse. If the acoustic energy is known, it is possible to obtain the acoustic efficiency for the air-bubble-stimulated underwater discharges. This is an important parameter which can be used in performance evaluation of the plasma-acoustic systems. The acoustic efficiency, $\eta_{a c}$, can be defined as a ratio of the acoustic energy in the pressure impulse to the total energy delivered into the plasma channel:

$$
\eta_{a c}=E_{a c} / E_{p l} \times 100 \%
$$

The energy delivered into the plasma, $E_{p l}$, can be obtained by equation (3). However, the exact calibration of the pressure sensor, Pinducer VP1093, used in the diagnostic system is not provided by the manufacturer. Therefore, an alternative way to obtain the pressure waveform $P_{a c}(t)$ was developed: an analytical model which linked the acoustic waveform $P_{a c}(t)$, to the hydrodynamic (the radius of the cavity, $r(t)$,) and the electrical parameters of the circuit. This model was used in order to calculate the profile of the acoustic impulse.

\subsubsection{ENERGY BALANCE EQUATION}

The developed analytical model is based on the energy balance equation which links the electrical energy, released in the plasma channel with the mechanical work done by the gasplasma channel and the internal energy of the plasma:

$$
P_{i} \frac{d\left(V_{c}\right)}{d t}+P_{\infty} \frac{d V_{c}}{d t}+\frac{1}{\gamma-1} \frac{d\left(P_{i} V_{c}\right)}{d t}=P_{e l t}
$$

where $P_{i}$ is the pressure inside the plasma channel, $V_{a c}$ is the volume of the cavity, $P_{\text {elt }}$ is the electrical power, $P_{e l t}=R_{p l} \times I(t)^{2}$, and $\gamma$ is the constant ratio of specific heats and the value of $\gamma=1.3$ is chosen according to [6].

The first two terms in equation (9) represent the mechanical work done by the expanding cavity against the water and the potential energy stored in the surrounding water respectively. The third term is the thermal (internal) energy of the gas/plasma in the cavity, which is considered to behave like an ideal gas.

\subsubsection{CAVITY DYNAMICS AND ACOUSTIC IMPULSE}

Gilmore's cavity oscillation model based on the Kirkwood Bethe hypothesis [28, 29] was used to describe the dynamic behavior of the cavity. During the initial energy deposition stage of the spark discharge, the gas/water interface of the formed cavity experiences an intense acceleration, potentially resulting in compression of the water in the vicinity of the plasma channel. Thus, a modified version of Tait's equation of the state for water, [30], and the pressure-dependent speed of sound in water, $c$, were used in the developed model:

$$
\frac{P_{i}+B}{P_{\infty}+B}=\left(\frac{\rho}{\rho_{\infty}}\right)^{n}, \mathrm{c}=\left(c_{\infty}^{2}+(n-1) H\right)^{\frac{1}{2}}
$$

where $P_{i}$ is the internal pressure of the formed cavity, $P_{\infty}$ is the constant hydrostatic pressure, $B=0.3 \mathrm{GPa}$ and $n=7$ for water; $\rho$ and $\rho_{0}$ are the density of the water with and without an external pressure disturbance respectively; $c_{\infty}$ is the local speed of undisturbed water and $H$ is the enthalpy of the water at the gas/water interface [22]:

$$
H=\frac{n}{n-1} \frac{\left(\rho_{\infty}+B\right)^{\frac{1}{n}}}{\rho_{\infty}}\left[\left(P_{i}+B\right)^{\frac{n-1}{n}}-\left(\rho_{\infty}+B\right)^{\frac{n-1}{n}}\right]
$$

Based on the hypotheses (10) and (11), Gilmore's model for the cavity's oscillation in a compressible liquid is given as:

$$
\left(1-\frac{\dot{r}}{c}\right) r \ddot{r}+\frac{3}{2}\left(1-\frac{\dot{r}}{3 c}\right) \dot{r}^{2}=\left(1+\frac{\dot{r}}{c}\right) H+\left(1-\frac{\dot{r}}{c}\right) \frac{r}{c} \dot{H}
$$

where $\dot{r}$ and $\ddot{r}$ are the first and second time derivatives of the cavity's radius, $r(t)$, respectively. Equation (12) describes the expansion and collapse of the discharge cavity.

The primary acoustic impulse, $P_{a c}(t)$, emitted by the expanding plasma channel is described by the following equation in the acoustic approximation:

$$
P_{a c}(t)=P_{\infty}+\rho_{\infty}\left(\frac{1}{d}\left(r^{2} \ddot{r}+2 r \dot{r}^{2}\right)-\frac{r^{4}}{2 d^{4}} \dot{r}^{2}\right)^{2}
$$

where $d=0.5 \mathrm{~m}$ in this study.

\subsubsection{SIMULATION REUSLTS}

Equations (9)-(13) were used in [5, 6], to model free underwater discharges, and it was confirmed that this approach satisfactorily describes the evolution of the cavity's radius. In this paper, equation (9)-(13) were solved in the Simulink (MatLab R2016a) environment for air-bubblestimulated discharges. Four initial parameters were used in the model, $I_{0}, R_{p l}, \alpha$ and $\omega$ these parameters were obtained from the experimental current waveforms for each individual discharge. These initial conditions together with the other constants used in this model are listed in Table 2.

It was found that the model was not very sensitive to the choice of the initial parameters; the maximum change in the 
acoustic output was only $\sim 4 \%$ for a $\sim 900 \%$ change in the initial values of the cavity radius and its velocity. The cavity dynamics and the acoustic parameters are defined by the energy input rather than by the initial values (initial cavity radius and velocity).

Table 2. Initial Conditions for the Model

\begin{tabular}{c|c}
\hline Parameter & Value \\
\hline$r(0)$ & $0.0001 \mathrm{~m}$ \\
$\dot{r}(0)$ & $0.001 \mathrm{~m} / \mathrm{s}^{2}$ \\
$d$ & $0.5 \mathrm{~m}$ \\
$P_{\infty}$ & $10^{5} \mathrm{~Pa}$ \\
$\rho_{\infty}$ & $10^{3} \mathrm{~kg} / \mathrm{m}^{3}$ \\
$c_{\infty}$ & $1483 \mathrm{~m} / \mathrm{s}$ \\
\hline
\end{tabular}

By knowing $P_{a c}(t)$, the acoustic energy, $E_{a c}$, was obtained by the model and the acoustic efficiency, $\eta_{a c}$, was calculated by using Equation (9) for all tests conducted in this paper. The relationship between $\eta_{a c}$ and $R_{p l}$, and between $\eta_{a c}$ and $E_{p l}$ are given in Figure 7.

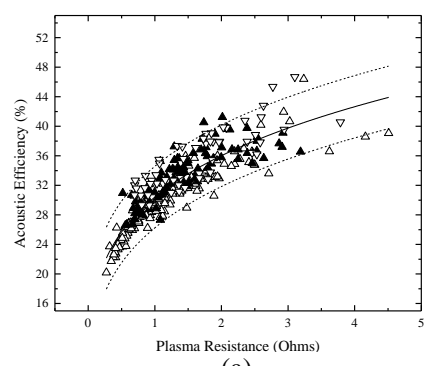

(a)

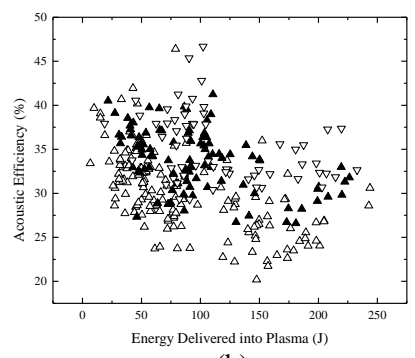

(b)
Figure 7. The acoustic efficiency as a function of (a) the plasma resistance; and (b) the energy delivered in plasma for all inter-electrode distances. Open upper triangles represent data for $\ell=15 \mathrm{~mm}$; closed triangle represent data for $\ell=20 \mathrm{~mm}$; open inverted triangles represent data for $\ell=25 \mathrm{~mm}$. Solid line indicates the fitting of equation (14), dashed lines demonstrate the $95 \%$ prediction bands.

Figure 7a shows the functional behavior of the acoustic efficiency $\eta_{a c}$ with the plasma resistance for all inter-electrode distances. It can be seen that $\eta_{a c}$ is in the range from $20 \%$ to $47 \%$, which is close to the values of the acoustic efficiency obtained in [31] for the free UWSD's, 23-30\%. $\eta_{a c}$ increases with an increase in $R_{p l}$ for all tested $\ell$ 's, however, $\eta_{a c}$ demonstrate variable sensitivity to the different inter-electrode gaps. The air-bubble-stimulated discharges with longer $\ell$ have higher plasma resistances, $R_{p l}$, thus the highest $\eta_{a c}$ was obtained for the $20 \mathrm{~mm}$ and $25 \mathrm{~mm}$ cases. However, $\eta_{a c}$ for $\ell$ $=15 \mathrm{~mm}$ concentrate at lower levels, Figure $7 \mathrm{~b}$ For the same value of $R_{p l}$ (Figure 7a), the discharges with longer $\ell$, especially for the $25 \mathrm{~mm}$ case, always generate higher $\eta_{a c}$ due to the enhanced values of $P_{a c-\max }$ for longer $\ell$ 's. Data in Figure 7 (a) can be fitted with a phenomenological scaling relation:

$$
\eta_{a c}=s R_{p l}^{x}
$$

where $s$ and $x$ are fitting coefficients, and $R_{p l}$ is in Ohms.

The fitting of Equation (14) to the experimental data is shown as a solid line in Figure $7 \mathrm{a}$; the dashed lines indicate the $95 \%$ prediction intervals. Coefficient $s$ was found to be $30.41 \pm 0.22$ and the power coefficient, $x$, is $0.24 \pm 0.01$. Equation (14) provides a satisfactory fitting as the $95 \%$ prediction interval includes most of the data points.

Figure $7 b$ shows the variation of the acoustic efficiency with the energy delivered into the plasma channel for all interelectrode distances. It can be seen that $\eta_{a c}$ decreases slowly with an increase in $E_{p l}$ which is confirmed by the finding that relative changes in $E_{p l}$ and $E_{a c}$, lead to a decrease in the energy ratio, $E_{a c} / E_{p l}$. Figure $7(\mathrm{~b})$ also shows that $\eta_{a c}$ is higher for longer $\ell$, for the same energy delivered into the plasma.

Based on the established dependency of $\eta_{a c}$ on $\ell, C, R_{p l}$ and $E_{p l}$, it can be concluded that the increase in $E_{a c}$, especially obtained by an increase in $C$ and $V_{b r}$, does not necessarily lead to an enhanced acoustic efficiency, $\eta_{a c}$. Therefore, it is not possible to achieve an increase in both, $E_{a c}$ and $\eta_{a c}$ by simply injecting more energy into the plasma.

The results shown in Figure 7 show that discharges with longer $\ell$ and lower $C$ provide higher acoustic efficiency as compared with discharges with shorter $\ell$ and higher $C$ (providing that these discharges produce the same acoustic peak magnitude). Thus, this study stresses the importance of the breakdown mechanism in the optimisation of acoustic parameters of the plasma-acoustic sources: air-bubblestimulated discharges can be developed in longer interelectrode gaps at lower energisation levels. This finding is important for different practical applications and allows for optimisation of both, acoustic magnitude and acoustic efficiency of UWSD's by selecting the appropriate combinations of the electrodes topology, energisation and circuit parameters.

\section{CONCLUSION}

A comprehensive investigation of the characteristics of the underwater spark discharges initiated by injecting air bubbles into the inter-electrode gap has been conducted. It was shown that in the case of air-bubble-initiated UWSD's the interelectrode gaps could be significantly increased compared to those for free discharges. The inter-electrode gap used in this study was increased from $15 \mathrm{~mm}$ to $25 \mathrm{~mm}$ under the same electrical parameters (capacitance and charging voltage) as used in the case of free discharges in [6]. The main advantage provided by longer inter-electrode gaps, is an increase in the peak magnitude of the emitted acoustic impulses and an increase in the acoustic efficiency.

Two phenomenological scaling relations, (4) and (5), were developed to describe the link between the acoustic and hydrodynamic parameters of the air-bubble-stimulated UWSD's, $P_{a c-m a x}$ and $T_{c a v}$, and $V_{b r}$. The increase in $C$ and $V_{b r}$ resulted in an increase in both, $T_{c a v}$ and $P_{a c-m a x}$ generated by these discharges. It was also shown that both, $T_{c a v}$ and $P_{a c-m a x}$ increase with an increase in the energy delivered into plasma, $E_{p l}$. These relationships are due to an inverse proportionality between $R_{p l}$ and $E_{p l}$, which was established in [8]. The 
relationship between $T_{c a v}$ and $E_{p l}$, and the link between $P_{a c-\max }$ and $E_{p l}$ are provided by equations (6).

The model which describes the dynamics of postbreakdown gas/plasma cavities and the acoustic impulses generated by these cavities was developed based on the Gilmore's bubble oscillation model in a compressible liquid, and the energy balance equation. The model provides a good agreement between the experimental and calculated values for the period of the cavity oscillation, $T_{c a v}$. The modelled acoustic impulses were used to calculate the acoustic efficiency, $\eta_{a c}$ of the air-bubble-stimulated UWSD's.

The dependencies of $P_{a c-\max }$, and $\eta_{a c}$ on $R_{p l}$ have been obtained and it was shown that for these experimental conditions $\eta_{a c}$ increases with an increase in plasma resistance. However, it was found that $\eta_{a c}$ decreases with an increase in the total energy delivered into the plasma. High values of $\eta_{a c}$ were obtained for either lower $E_{p l}$ or longer $\ell$. Therefore, the optimal conditions to achieve high $\eta_{a c}$ and high $P_{a c \text {-max }}$ should include the use of lower capacitance in the pulsed power circuit, $C$, together with a longer inter-electrode distance. These requirements are difficult to achieve in free discharges in conducting water therefore the stimulation of the development of the discharges, for example by injecting air bubbles, in order to achieve successful breakdown in longer inter-electrode gaps is required. Also, air-bubble-stimulated discharges allows for higher pulse repetition rates as compared with the wire-guided discharges, [8]. The results obtained in this study will help in the optimisation of the plasma acoustic sources and in tailoring of the acoustic wave-forms generated by the underwater spark discharges.

\section{REFERENCES}

[1] M. Wilson, L. Balmer, M. Given, S. MacGregor, I. Timoshkin, “An investigation of spark discharge parameters for material processing with high power ultrasound", Min. Eng., vol. 20, pp. 1159-1169, 2007.

[2] K. Chung, S. Lee, Y. Hwang, C. Kim, "Modeling of pulsed spark discharge in water and its applications to well cleaning", Curr. Appl. Phys., vol. 15, pp. 977-986, 2015.

[3] T. H. Dang, A. Denat, O. Lesaint, G. Teissedre, "Pulsed electrical discharges in water for removal of organic pollutants: a comprehensive study", Eur. J. Appl. Phys., vol. 47, no. 22818, 2009.

[4] http://woodshole.er.usgs.gov/operations/sfmapping/sparker.htm.

[5] I. Timoshkin, R. Fouracre, M. Given, S. MacGregor, "Hydarodynamic modeling of transient cavities in fluids generated by high voltage spark discharges", J. Phys. D: Appl. Phys., vol. 39, pp. 4808-4817, 2006.

[6] J. W. Mackersie, I. V. Timoshkin and S. J. MacGregor, "Generation of high-power ultrasound by spark discharge in water", IEEE Trans. on Plasma Sci., vol. 33,no. 5, pp. 1715-1724, 2005.

[7] Y. Sun, I. Timoshkin, M. Given, M. Wilson, T. Wang, S. J. MacGregor, "Impulsive discharges in water: acoustic and hydrodynamic parameters," IEEE Trans. on Plasma Sci., vol. 44, pp. 2156-2166, 2016.

[8] Y. Sun, I. Timoshkin, M. Given, M. Wilson, T. Wang, S. J. MacGregor, "Electrical and Acoustic Paramters of Wire-Guided Discharges in Water: Experimetnal Determination and Phenomenological Scaling", IEEE Trans. on Plasma Sci., vol. 45, pp. 2648-2655, 2017.

[9] R. M. Roberts, J. A. Cook, R. L. Rogers, "The energy partition of underwater sparks", J. Acoust. Soc. Amer., vol. 99, No. 6, pp. 34653475, 1996.

[10] I. Timoshkin, S. MacGregor, M. Given, R. Fouracre, "High power ultrasound impulses induced by wire-guided spark discharges in water", IEEE $16^{\text {th }}$ Int. Pulsed Power Conf., 2007, pp. 33-36.
[11] M. Zahn, S. Voldman, T. Takada, D. Fenneman, "Charge injection and transport in high voltage water/glycol capacitors", J. Appl. Phys., vol.54, pp.315-325, 1983.

[12] M. Zahn, Y. Ohki, K. Rhoads, M. LaGasse, H. Matsuzawa, "ElectroOptic Charge Injection And Transport Measurements In Highly Purified Water And Water/Ethylene Glycol Mixtures", IEEE Electr. Insul., vol. EI-20, no.2, pp.199-211, 1985.

[13] M. Zahn, Y. Ohki, D. Fenneman, R. Gripshover, V. Gehman, "Dielectric Properties of Water and Water/Ethylene Glycol Mixtures for Use in Pulsed Power System Design”, Proc. of the IEEE, vol. 74, no.9, pp.1182- 1221, 1986.

[14] A. Hamdan, M. S. Cha, "The effects of gaseous bubble composition and gap distance on the characteristics of nanosecond discharges in distilled water", J. Phys. D: Appl. Phys., vol. 49, no. 24, 2016.

[15] V. Stelmashuk, "Microsecond electrical dischagre in water in plate-toplate configuration with nitrogen bubble injection", IEEE Trans. on Plasma Sci., vol. 44, no.4, pp 702-707, 2016.

[16] N. Y. Babaeva, D. V. Tereshonok, G. V. Naidis, "Initiation of breakdown in bubbles immersed in liquids: pre-existed charges versus bubble size", J. Phys. D: Appl. Phys., vol. 48, no. 35, 2016.

[17] B. S. Sommers and J. E. Foster, "Plasma formation in underwater gas bubbles", J. Phys. D: Appl. Phys., vol. 23, no. 1, 2014.

[18] Y. Hayashi, N. Takada, H. Kanda, M. Goto, "Effect of fine bubbles on electrical discharge in water", Plasma Source Sci., vol. 24, no. 5, 2015.

[19] Voltage Probe Manual and Data North Star High Voltage, Inc. Rev January 2016, http://www.highvoltageprobes.com/high-voltage-probe

[20] I.V. Timoshkin J.W. Mackersie, S.J. MacGregor, "Plasma channel miniature hole drilling technology", IEEE Trans.Plasma Science., vol. 32, no. 5, pp. 2055-2061, 2004.

[21] S. Buogo, J. Plocek, K. Vokurka, "Efficiency of energy conversion in underwater spark discahrges and associated bubble oscillations: experimental results", ACTA, vol. 95 46-59, pp. 46-59, 2009.

[22] C. E. Brennen, Fundamentals of Multiphase Flows, Cambridge University Press, Pasadena California, Ch. 4, 2005.

[23] L. A. Crum, T. J. Mason, J. L. Reisse, Sonochemistry and Sonoluminescence, Kluwer Academic Publishers, New Haven, pp. 183190, 1999.

[24] Y. Liu, Y. Z. Li, D. X. Li, W. S. Liu, Y. G. Zhou, C. F. Lin, "Intensity improvement of shock waves induced by liquid electrical discharges", Phys. of Plasmas, vol. 24, no. 043510, 2017.

[25] M. G. Danikas, "Breakdown of Transformer Oil", IEEE Electr. Insul. Mag., vol. 6, no. 5, pp.27-34, 1990.

[26] A. Jaksts, J. D. Cross, "High Speed Streak Photography Of The Breakdown Of Transformer Oil And The Dependence Of Its Nature On Local Stored Energy", IEEE Trans. Electr. Insul, vol. El-18, no.6, pp.599-604, 1983.

[27] J. D. Cross, "A physical explanation of the effects of electrode area on the breakdown of liquid dielectrics", Can. Elec. Eng. J., vol 7, no 2, pp.28-30, 1982.

[28] T. G. Leighton, The Acoustic Bubble, Academic Press Inc., London, Ch. 4, 1994.

[29] A. Ziolkowski, "Measurement of air-gun bubble oscillation", Geophysics, vol. 63, no. 6, pp. 2009-2024, 1998.

[30] F. R. Gilmore, "The growth or collaspe of a shperical bubble in a viscous compressble liquid”, Hydrodynamics Lab., Report No. 26-4, 1952.

[31] Y. Liu, Y. Z. Li, D. X. Li, Y. G. Zhou, H. Li, Q. Zhang, C. F. Lin, "Energy transfer efficiency improvement of liquid pulsed current discharge by plasma channel length reualgtion mode", IEEE Trans. Plasma Sci., vol.45, no. 12, pp. 3231 - 3239, 2017.

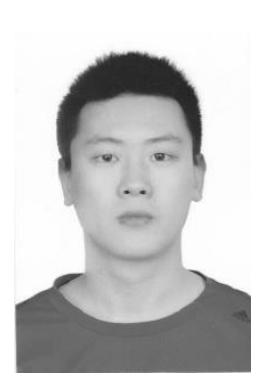

Ying Sun was born in Shandong, China. $\mathrm{He}$ received the B.Eng degree in electronic and electrical engineering in 2012 and M.Sc degree in electrical power engineering with business in 2013 from University of Strathclyde, Scotland, 
United Kingdom. He is currently pursuing the Ph.D degree in high voltage technology at University of Strathclyde, Scotland, UK.

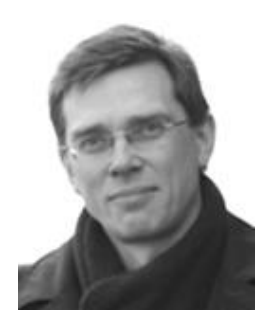

Igor V. Timoshkin (M'07-SM'14) received a degree in physics from the Moscow State University (Russia) in 1992, and the Diploma and the Ph.D. degree from the Imperial College of Science, Technology and Medicine, (London, UK) in 2001. After graduation from MSU he worked as a Researcher at Moscow State Agro-Engineering University, and then at the Institute for High Temperatures of Russian Academy of Sciences before moving to ICSTM in 1997. He joined the Department of Electronic and Electrical Engineering of the University of Strathclyde (Glasgow, UK) in 2001 where he became a Reader in 2016. His research interests include transient discharges in condensed media, practical applications of electro-hydraulic and high-power ultrasound pulses, biodielectrics and effects of electromagnetic fields on biological objects.

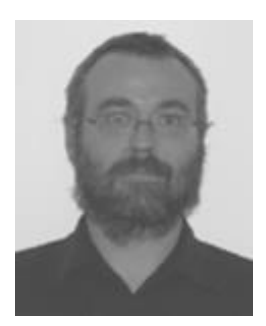

Martin J. Given (M'99-SM'11) is currently a Senior Lecturer in the Department of Electronic and Electrical Engineering at the University of Strathclyde. He received a degree in physics from the University of Sussex in 1981 and $\mathrm{ahD}$ in electronic and electrical engineering from the University of Strathclyde in 1996. His research interests include, ageing processes and condition monitoring in solid and liquid insulation systems, high speed switching and pulse power.

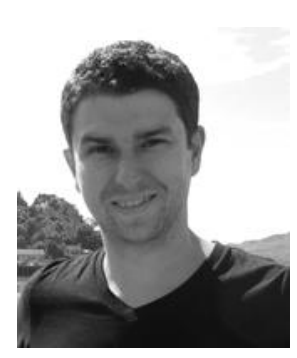

Mark P. Wilson (M'10) was born in Stranraer, Scotland, in 1982. He received the B.Eng. (with honours), M.Phil., and Ph.D. degrees in electronic and electrical engineering from the University of Strathclyde, Glasgow, U.K., in 2004, 2007, and 2011, respectively. He is presently based in the High Voltage Technologies research group at the University of Strathclyde, where his research interests include interfacial surface flashover, nanodielectrics, and the practical applications of high power ultrasound, corona discharges, and pulsed electric fields. Mark is a member of the IEEE Nuclear and Plasma Sciences Society, from whom he received a Graduate Scholarship Award in 2011, the IEEE Dielectrics and Electrical Insulation Society, and the IET.

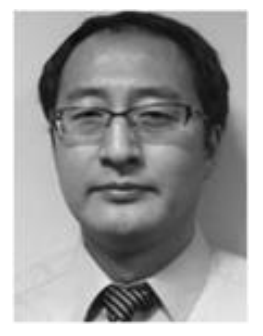

Tao Wang received the B.Eng and M.Sc degrees from Northeast China Dianli University (China) in 1993 and 1996 respectively, and the $\mathrm{Ph} . \mathrm{D}$. degree from the University of Strathclyde (Glasgow, UK) in 2005. He then joined the Newland Entech (China) as a research fellow developing high efficiency industrial ozone generator. He joined the department of Electronic and Electrical Engineering of University of Strathclyde as a lecturer in 2010. His research interests include non-thermal gas discharges and their applications in gas synthesis, water disinfection and advanced

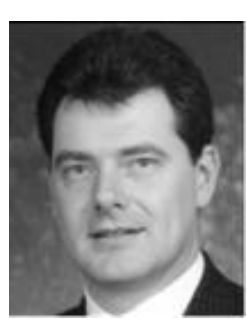
oxidation process in water.

\section{Scott J. MacGregor (M'95-SM'14)} received the B.Sc. and Ph.D. degrees from the University of Strathclyde, Glasgow, U.K., in 1982 and 1986, respectively. He became a Pulsed Power Research Fellow in 1986 and a Lecturer in pulsed-power technology in 1989. In 1994, he became a Senior Lecturer, with a promotion to Reader and Professor of High Voltage Engineering, in 1999 and 2001, respectively. In 2006 and 2010 he became Head of the Department of Electronic and Electrical Engineering and Executive Dean of the Faculty of Engineering, and has been the Vice-Principal of the University of Strathclyde since 2014. Professor MacGregor was the recipient of the 2013 IEEE Peter Haas Award, and he was appointed as an Associated Editor of the IEEE Transitions on Dielectrics and Electrical Insulation in 2015. His research interests include high-voltage pulse generation, high-frequency diagnostics, high-power repetitive switching, high-speed switching, electronic methods for food pasteurization and sterilization, generation of high-power ultrasound (HPU), plasma channel drilling, pulsed-plasma cleaning of pipes, and stimulation of oil wells with HPU.

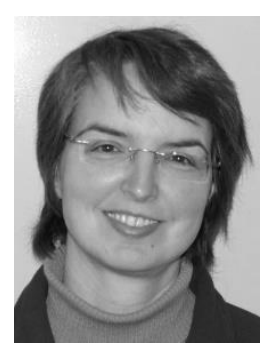

Nelly Bonifaci graduated from University Joseph Fourier with a MEng degree in Physics in 1989. In 2008 she obtained the HDR Habilitation qualification from the University of $\mathrm{J}$ Fourier, Grenoble. Dr Bonifaci joined the Laboratory on Dielectric Materials and Electrostatics (now G2E laboratory) CNRS, France in 1989 where she became a $1^{\text {st }}$ class researcher in 1997. In 2007 she was promoted to a position of a Senior Researcher/Team Leader. Dr Bonifaci research interests include breakdown in insulating liquids, plasma streamers in liquid dielectrics, pre-breakdown and plasma phenomena in dielectric liquids including corona discharges and electronic transport. Dr Bonifaci is an Editor of the IEEE Transactions of Dielectric and Electrical Insulation and a member of International Organising Committee of the IEEE Conference on Dielectric Liquids. 